\title{
HERMES high-resolution spectroscopy of HD 149382 Where did the planet go?
}

\author{
V.A. Jacobs*, R.H. Østensen*, H. Van Winckel*, S. Bloemen*, P.I. Pápics*, \\ G. Raskin*, J. Debosscher*, S. Uttenthaler*, E. Van Aarle*, C. Waelkens*, \\ E. Bauwens*, T. Verhoelst*, C. Gielen*, H. Lehmann ${ }^{\dagger}$ and R. Oreiro*** \\ ${ }^{*}$ Instituut voor Sterrenkunde, Katholieke Universiteit Leuven, Celestijnenlaan 200D, B-3001 \\ Leuven, Belgium \\ ${ }^{\dagger}$ Thüringer Landessternwarte Tautenburg, Karl-Schwarzschild-Observatorium, 07778 Tautenburg, \\ Germany \\ **Instituto de Astrofísica de Andalucía-CSIC, E-18008 Granada, Spain
}

\begin{abstract}
A close substellar companion has been claimed to orbit the bright sdB star HD 149382 with a period of $2.391 \mathrm{~d}$. In order to check this important discovery we gathered 26 high resolution spectra over 55 days with the HERMES spectrograph on the $1.2 \mathrm{~m}$ Mercator telescope on La Palma, and analyzed the resulting radial velocities. Our data show no sign of any significant radial-velocity periodicities, and from the high precision of our measurements we rule out any RV variations with amplitudes higher than $0.79 \mathrm{~km} / \mathrm{s}$ on periods shorter than 50 days.
\end{abstract}

Keywords: Substellar companions; planets

PACS: $97.82 . \mathrm{Fs}$

\section{INTRODUCTION}

HD 149382 was recognised to be a subdwarf B star by MacConnell and Frye (1972), and the spectroscopic analysis of Saffer et al. (1994) place it on the hot end of the extreme horizontal branch (EHB). With a magnitude of $\mathrm{V}=8.9$ it is the brightest EHB star in the sky, and one of just a handful of such subdwarfs that can be easily observed with high-resolution spectroscopy on 1-m class telescopes. The first hints of a binary nature for HD 149382 came with the study of Ulla and Thejll (1998), where they used infrared JHK colours as an indicator of binarity, and concluded that HD 149382 has a very signficant IR excess, compatible with a companion of class K1 to G2. Such binaries are predicted to form via stable Roche lobe overflow, and result in orbital periods of hundreds of days (Han et al. 2003). A red companion was clearly detected about 1" away from the subdwarf in high resolution $\mathrm{H}$-band imaging obtained with the NAOMI adaptive optics system at the William Herschel Telescope on La Palma, by Østensen et al. (2005) (see Fig. 1). At $\sim 75 \mathrm{pc}$, this separation corresponds to $\sim 75$ AU making it too wide for the two stars to have interacted during the evolution of the primary. There is also no significant change in the separation between the images obtained in 2002 and 2004, so any orbital period must be on the order of decades, if these objects are gravitationally bound at all.

Later, a close companion was claimed to be found around HD 149382 by Geier et al. 

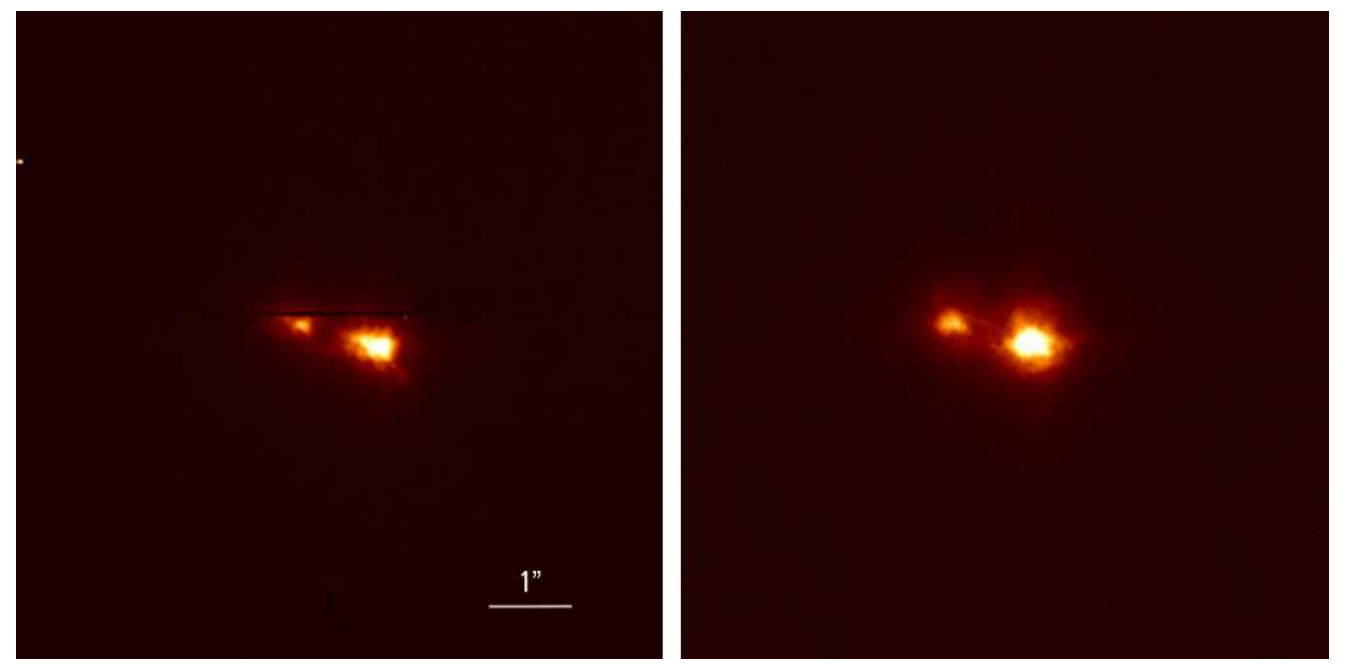

FIGURE 1. A red companion was clearly detected about 1 " away from the subdwarf in high resolution H-band imaging obtained with the NAOMI adaptive optics system at the William Herschel Telescope on La Palma, by Østensen et al. (2005)

(2009), based on 15 high-resolution spectra $(R=30000-48000)$ on three different spectrographs (ESO-2.2m/FEROS, CAHA-2.2m/FOCES, and McDonald-2.7m/Coudé) taken within four years and one additional VLT/UVES spectrum $(R=80000)$. This was also the first claim that a close substellar companion, able to influence the stellar evolution of the host star, was found, thus challenging evolutionary experts to come up with a theory in which single stars with substellar companions were able to produce a subdwarf. This led to the profound question of what the actual influence of the companion might be, as such a low mass companion would be able to aid the formation of a hot subdwarf.

\section{OBSERVATIONS}

We gathered 26 high-resolution spectra over a timebase of 55 days (Table 2) with the HERMES spectrograph on the 1.2-m Mercator telescope on La Palma. HERMES reaches a spectral resolution of $\sim 85000$ and a spectral coverage from 377 to $900 \mathrm{~nm}$ in a single exposure with a peak efficiency of $28 \%$. The spectrograph is bench-mounted and fibre-fed, and resides in a temperature and pressure controlled enclosure to guarantee instrumental stability. The high resolution fibre has a sky aperture of $2.5 \mathrm{arcsec}$. The red companion to HD 149382 (see Fig. 1) is therefore close enough to enter the aperture and slightly contaminate the spectrum of the target.

Some spectra were taken during the early testing of the spectrograph. For three spectra, a problem occurred during integration and the exposure was read out early. The resulting spectra were of too low signal-to-noise to be useful, leaving us with 23 spectra to analyse. Four HeI lines at $6678.15 \AA$, $5015.68 \AA, 4713.14 \AA$ and 5875.6251 $\AA$, were used to determine the radial velocities by simultaneous fitting using the software 
TABLE 1. Overview of the observations of HD 149382. The first two rows refer to the high resolution infrared imaging with the NAOMI adaptive optics system at the William Herschel Telescope on La Palma, while the other rows refer to the high resolution HERMES spectra, taken with the Mercator telescope at La Palma. For completeness, the number of exposures, the exposure times and the observers are listed.

\begin{tabular}{lrrrr}
\hline Date & Telescope/Instrument & $\mathrm{N}_{\exp }$ & $\mathrm{T}_{\exp }[s]$ & Observer \\
\hline $2002 / 08 / 16$ & WHT/NAOMI/INGRID & 14 & 5.0 & R.H. $\varnothing$ \\
$2004 / 06 / 29$ & WHT/NAOMI/INGRID & 2 & 3.0 & R.H. \\
\hline $2009 / 06 / 30$ & Mercator/HERMES & 1 & 1500 & R.O. \\
$2009 / 06 / 30$ & Mercator/HERMES & 1 & 547 & J.D. \\
$2009 / 07 / 12$ & Mercator/HERMES & 1 & 900 & S.U. \\
$2009 / 07 / 13--18$ & Mercator/HERMES & 9 & 1800 & E.v.A. \\
$2009 / 07 / 21$ & Mercator/HERMES & 1 & 1800 & C.W. \\
$2009 / 07 / 31$ & Mercator/HERMES & 1 & 900 & E.B. \\
$2009 / 08 / 06--13$ & Mercator/HERMES & 8 & $1 \times 615,1 \times 900,6 \times 1800$ & T.V. \\
$2009 / 08 / 14--15$ & Mercator/HERMES & 2 & 1800 & C.G. \\
\hline
\end{tabular}

TABLE 2. Overview of the 22 radial velocities of HD 149382 as derived from the HERMES spectra.

\begin{tabular}{rrrr}
\hline Date & $\mathbf{R V}[\mathbf{k m} / \mathbf{s}]$ & Date & $\mathbf{R V}[\mathbf{k m} / \mathbf{s}]$ \\
\hline 2455003.45024 & $26.68 \pm 0.15$ & 2455034.45301 & $26.28 \pm 0.13$ \\
2455025.49846 & $26.09 \pm 0.19$ & 2455050.41444 & $25.13 \pm 0.22$ \\
2455026.40797 & $26.05 \pm 0.11$ & 2455051.38909 & $26.15 \pm 0.11$ \\
2455027.41298 & $26.42 \pm 0.10$ & 2455051.51929 & $26.09 \pm 0.11$ \\
2455027.55091 & $26.48 \pm 0.11$ & 2455055.41469 & $25.75 \pm 0.19$ \\
2455028.43085 & $26.36 \pm 0.10$ & 2455055.53976 & $26.37 \pm 0.10$ \\
2455028.59094 & $25.96 \pm 0.13$ & 2455056.38705 & $26.33 \pm 0.14$ \\
2455029.40622 & $26.21 \pm 0.11$ & 2455056.43345 & $26.48 \pm 0.13$ \\
2455030.41048 & $26.16 \pm 0.11$ & 2455057.41243 & $26.61 \pm 0.17$ \\
2455030.50488 & $25.93 \pm 0.12$ & 2455058.38674 & $26.70 \pm 0.11$ \\
2455031.41011 & $26.38 \pm 0.11$ & 2455058.51017 & $26.44 \pm 0.14$ \\
\hline
\end{tabular}

package Molly ${ }^{1}$. One spectrum resulted in an error of more than $2 \mathrm{~km} / \mathrm{s}$ and as this was much higher than the other errors (at a $3 \sigma$-level), it was deleted from the list. 22 useful spectra remain in Table. 2.

\section{RESULTS}

Fig. 3 shows the amplitude spectrum of our HERMES observations, and the associated spectral window. Clearly no significant frequencies can be found, nor do we find similar amplitudes to those reported by Geier et al. (2009).

\footnotetext{
${ }^{1}$ We thank Tom Marsh for the use of 'molly'.
} 

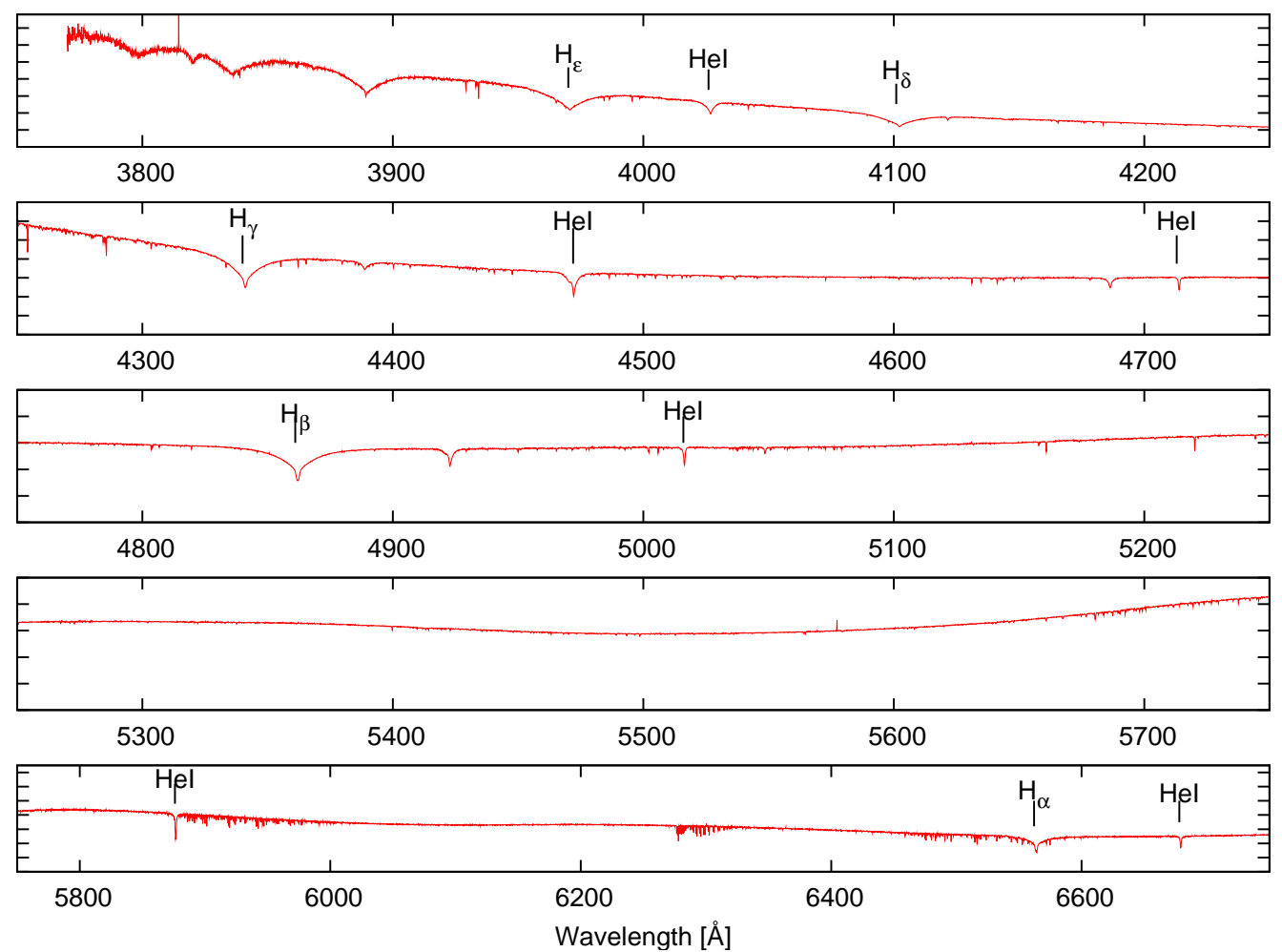

FIGURE 2. Median spectrum of 22 observations of HD 149382. The four helium lines, that were used for the determination of the radial velocities are indicated.
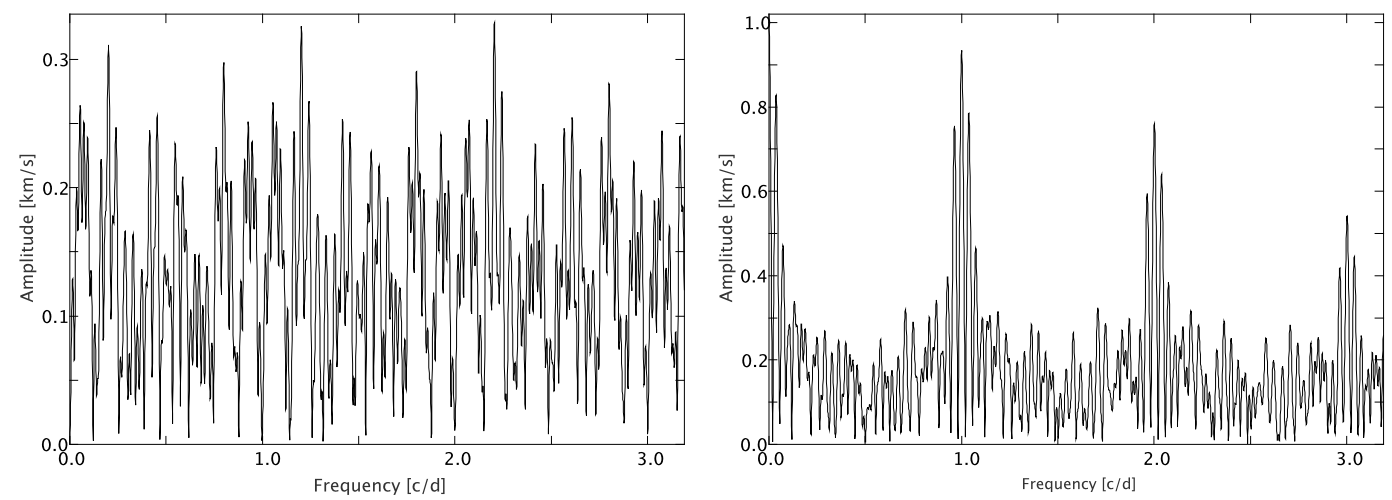

FIGURE 3. Left panel: The amplitude spectrum of our HERMES observations. Clearly, no significant frequencies can be found, nor do we find similar amplitudes to those reported by Geier et al. (2009). Right panel: The spectral window of our HERMES observations clearly shows the 1 day alias.

\section{CONCLUSIONS}

Our additional data clearly shows that there is as yet no evidence for a close substellar companion surrounding the bright sdB star HD 149382. We gathered 22 spectra with the HERMES spectrograph on the Mercator Telescope. Our data show no sign of any radial- 


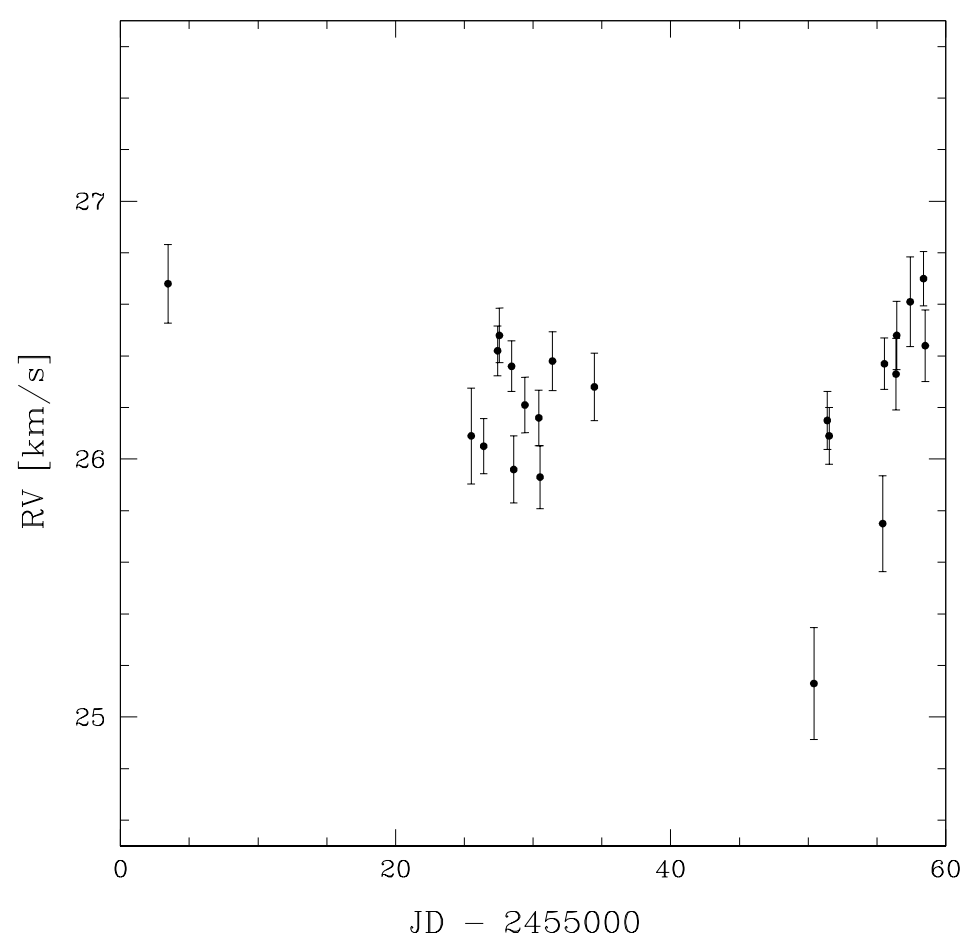

FIGURE 4. Radial velocities of HD 149382 as derived from the HERMES spectra.

velocity periodicities, and from the high precision of our measurements we rule out any RV variations with amplitudes higher than $0.79 \mathrm{~km} / \mathrm{s}$ on periods shorter than 50 days.

\section{ONLINE DATA}

The extracted spectra used in this work are available on-line at http://www. ster. kuleuven.be/instruments/hermes/data/HD149382/.

\section{REFERENCES}

D. J. MacConnell, and R. L. Frye, Publications of the Astronomical Society of the Pacific 84, 388 (1972). R. A. Saffer, P. Bergeron, D. Koester, and J. Liebert, Astrophysical Journal 432, 351 (1994).

A. Ulla, and P. Thejll, Astronomy and Astrophysics 132, 1 (1998).

Z. Han, P. Podsiadlowski, P. F. L. Maxted, and T. R. Marsh, Monthly Noticies of the Royal Astronomical Society 341, 669 (2003), arXiv:astro-ph/0301380.

R. Østensen, U. Heber, and P. Maxted, "Resolving sdB Binary Systems with Adaptive Optics," in 14th European Workshop on White Dwarfs, edited by D. Koester \& S. Moehler, 2005, vol. 334 of Astronomical Society of the Pacific Conference Series, 435.

S. Geier, H. Edelmann, U. Heber, and L. Morales-Rueda, Astrophysical Journal 702, L96 (2009), arXiv:0908.1025. 